\title{
Application of Optimal Homotopy Asymptotic Method for Solving Linear Boundary Value Problems Differential Equation
}

\author{
Malik . S. Awwad, Osama . Y Ababneh \\ Department of Mathematics, Faculty of Science \\ Zarqa University, Zarqa, Jordan \\ E-mail: awwadmalik@yahoo.com \\ E-mail: osababneh@zu.edu.jo
}

\begin{abstract}
The objective of this study are to apply the OHAM to find approximate solutions of singular two-point boundary value problems comparisons with exact solutions and other method like spline method were made. The results of equations studied using OHAM solutions were significantly reliable.
\end{abstract}

Keywords: Boundary value problems, optimal homotopy asymptotic.

2000 MSC No: 34K28, 35A24, 35F30.

\section{Introduction}

Boundary value problems (BVPs) ply a significant role in various of mathematical modeling, such as, physical methods, most phenomena occurring nonlinear and described by nonlinear equations, so solving the nonlinear equation has been a main focus. The optimal homotopy asymptotic methods (OHAM) one of the modeling used to solve linear and nonlinear differential equation. Was presented firstly by Marinca et al. [8-10] aiming at solving nonlinear problems without depending on a small parameter. generalization and reliability of this method were proved and solutions of currently important applications in science and engineering were obtained by several authors [1-7]. It can be noted that the HAM and HPM are special cases affiliated to OHAM. An of advantages of OHAM is that it does not require the identification of the curve and it is also parameter free.

In OHAM, the control and adjust of the convergence region are provided in a convenient way. Moreover, the OHAM has been built on convergence criteria similar to HAM but it differs from it in 
that its level of flexibility is greater than that of HAM [6]. This method is successfully applied by Marinca et al. $[9,10]$ to problems in mechanics, and has also shown its effectiveness and accuracy.

In this work, OHAM is applied successfully for finding approximate analytic solution of linear (BVPs), in section 2, we describe the basic idea of OHAM, in section 3, two examples are presented to illustrate the sufficiency of method, and the conclusion of this study is presented in the last section.

\section{Analysis of Method}

Consider the following differential equation

$$
L(u(x))+g(x)+N(u(x))=0, B\left(u, \frac{d u}{d x}\right)=0 .
$$

Where $L$ is the linear operator, $N$ nonlinear operator, $u(x)$ is an unknown function, $x$ denotes an independent variable, $g(x)$ is a known function and $B$ is a boundary operator.

By means of OHAM one first constructs a family of equations

$$
\begin{aligned}
& \begin{aligned}
(1-q)[L(v(x ; q))+g(x)] \\
\quad=H(q)[L(v(x ; q))+g(x)+N(v(x, q))],
\end{aligned} \\
& B\left(v(x ; q), \frac{d v(x ; q)}{d x}\right)=0,
\end{aligned}
$$

where $q \in[0,1]$ is an embedding parameter, $H(q)$ is a nonzero auxiliary function for $q \neq 0$ and $H(0)=0, v(x, q)$ is an unknown function.

Obviously, when $q=0$ and $q=1$ it holds that $v(x, 0)=u_{0}(x)$ and $v(x, 1)=u(x)$ 
respectively. Thus, as $q$ varies from 0 to 1 , the solution $v(x, q)$ approaches form $u_{0}(\mathrm{x})$ to $u(\mathrm{x})$ where $u_{0}(\mathrm{x})$ is obtained from Eq. (3.1.2) for $q=0$.

$L\left(u_{0}(x)\right)+g(x)=0, B\left(u_{0}, \frac{d_{u_{0}}}{d x}\right)=0$.

Next, we choose the auxiliary function $H(q)$ in the form

$H(q)=q c_{1}+q^{2} c_{2}+q^{3} c_{3}+\ldots$,

where $c_{1}, c_{2}, c_{3}, \ldots$ are the convergent control parameters which can be determined later.

To get an approximate solution, we expand $v\left(x, q, c_{i}\right)$ in Taylor's series about $\mathrm{q}$ in the following manner,

$v\left(x, q, c_{i}\right)=u_{0}(x)+\sum_{k=1}^{\infty} u_{k}\left(x, c_{1}, c_{2}, \ldots, c_{k}\right) q^{k}$.

Substituting (5) into (2) and equating the coefficient of like powers of $q$, we obtain the following linear equation.

Zeroth order problem is given by Eq. (3) and the first order problem is given by Eq. (6)

$L\left(u_{1}(x)\right)+g(x)=c_{1} N_{0}\left(u_{0}(x)\right), B\left(u_{1}, \frac{d u_{1}}{d x}\right)=0$

and the second order problem is given as the follow:

$L\left(u_{2}(x)\right)-L\left(u_{1}(x)\right)=c_{2} N_{0}\left(u_{0}(x)\right)+c\left[L\left(u_{1}(x)\right)+N_{1}\left(u_{0}(x)\right)\right]$

, $u_{1}(x), B\left(u_{2}, \frac{d u_{2}}{d x}\right)$ 
The general governing for $u_{k}(x)$ are given by:

$$
\begin{aligned}
L\left(u_{k}(x)\right)- & L\left(u_{k-1}(x)\right) \\
& =\mathrm{c}_{k} N_{0}\left(u_{0}(x)\right) \\
& +\sum_{i=1}^{k-1} \mathrm{c}_{i}\left[L\left(u_{k-i}(x)+N_{k-i}\left(u_{0}(x), u_{1}(x), \ldots, u_{k-1}(x)\right)\right]\right. \\
& , \mathrm{B}\left(u_{k}, \frac{\mathrm{d} u_{k}}{\mathrm{~d} x}\right)=0 .
\end{aligned}
$$

Where $k=2,3, \ldots$, and $N_{m}\left(u_{0}(x), u_{1}(x), \ldots, u_{m}(x)\right)$ is the coefficient of $q^{m}$ in the expansion of $N(v(x ; q))$ about the embedding parameter q.

$$
\begin{aligned}
& N\left(v\left(x, q, c_{i}\right)\right)= \\
& N_{0}\left(u_{0}(\mathrm{x})\right)+\sum_{\mathrm{m}=1}^{\infty} N_{m}\left(u_{0}((x)), u_{1}(\mathrm{x}), \ldots, u_{\mathrm{m}}(\mathrm{x})\right) q^{m} .
\end{aligned}
$$

It has been observed that the convergence of the series (5) depends upon the auxiliary convergent control parameters $\mathrm{c}_{1}, \mathrm{c}_{2}, \mathrm{c}_{3}, \ldots$. If it is convergent at $q=1$, one has

$v\left(x ; c_{i}\right)=u_{0}(x)+\sum_{k=1}^{\infty} u_{k}\left(x, c_{1}, c_{2}, \ldots, c_{k}\right)$.

The result of the $\mathrm{m}$ th-order approximation is

$$
\tilde{u}\left(x, c_{1}, c_{2}, \ldots, c_{m}\right)=u_{0}(x)+\sum_{m=1}^{m} u_{i}\left(x, c_{1}, c_{2}, \ldots, c_{i}\right) .
$$

Substituting (11) into (1) yields the following residual

$$
\begin{aligned}
& R\left(x, c_{1}, c_{2}, \ldots, c_{m}\right)=L\left(\tilde{u}\left(x, c_{1}, c_{2}, \ldots, c_{m}\right)\right)+g(x) \\
& +N\left(\tilde{u}\left(x, c_{1}, c_{2}, \ldots, c_{m}\right)\right) .
\end{aligned}
$$


If $R=0$, then $\tilde{u}$ will be the exact solution. Generally, it does not happen, especially in nonlinear are problems.

In order to find the optimal values of $c_{i}, i=1,2,3, \ldots$, we first construct the functional.

$\mathrm{J}\left(\mathrm{c}_{1}, \mathrm{c}_{2}, \ldots, \mathrm{c}_{m}\right)=\int_{\mathrm{a}}^{\mathrm{b}} R^{2}\left(x, \mathrm{c}_{1}, \mathrm{c}_{2}, \ldots, \mathrm{c}_{m}\right) \mathrm{d} x$,

and the minimizing it, we have

$\frac{\mathrm{dj}}{\mathrm{dC}_{1}}=\frac{\mathrm{dj}}{\mathrm{dC}_{2}}=\cdots=\frac{\mathrm{dj}}{\mathrm{dC}_{m}}=0$.

Where $a$ and $b$ are in the domain of the problem. With these constants knows, the approximate solution (of order) is well determined.

\section{Application}

Example1: consider the Bessel's equation of order zero taken from Kanth, Ravi and Reddy (2005).

$$
u^{\prime \prime}(x)+\left(\frac{1}{x}\right) u^{\prime}(x)+u(x)=0, u^{\prime}(0)=0, u(1)=1 \text {. }
$$

The exact solution of this problem in the case is given by

$u(x)=\frac{J_{0}(x)}{J_{0}(0)}$

To use the basic idea of OHAM formulated and according to eq. (1), we define the linear and nonlinear operators in the following form:

$$
\begin{aligned}
& L(v(x ; q))=\frac{d^{2} v(x ; q)}{d x^{2}} . \\
& N(v(x ; q))=\frac{x d^{2} v(x ; q)}{d x^{2}}+\frac{d v(x ; q)}{d x}+x v(x ; q) .
\end{aligned}
$$


Now, apply eq. (3) when $q=0$, it gives the zeroth-order problem as follow:

$u^{\prime \prime}(0)=0, u_{0}(1)=1, u_{0}{ }^{\prime}(0)=0$

The solution of eq. (4) is given by

$u_{0}(x)=0$.

From eq. (6), the first-order problem is

$u_{1}{ }^{\mathrm{I}}\left(x, c_{1}\right)=\mathrm{c}_{1}, u_{1}{ }^{\prime}(0)=0, u_{1}(1)=0$.

This has the following solution

$u_{1}\left(x, c_{1}\right)=\frac{1}{2}\left(-1+x^{2}\right) c_{1}$

According to eq. (7), the second-order problem is

$u_{2}{ }^{\prime \prime}\left(x, c_{1} c_{2}\right)=c_{2}+c_{1} u_{1}(x)+\frac{c_{1} u_{1}{ }^{\prime}(x)}{x}+u_{1}{ }^{\prime \prime}(x)+c_{1} u_{1}{ }^{\prime \prime}(x)$,

$u_{2}{ }^{\prime}(0)=0, u_{2}(1)=0$.

And has the solution

$u_{2}\left(x, c_{1}, c_{2}\right)=\frac{1}{24}\left(-1+x^{2}\right)\left(12 c_{1}+\left(19+x^{2}\right) c_{1}^{2}+12 c_{2}\right)$.

By applying equation (8) for $k=3$, the third-order problem is defined as:

$u_{3}{ }^{\prime \prime}\left(x, c_{1}, c_{2}, c_{3}\right)=\mathrm{c}_{3}+\mathrm{c}_{2} u_{1}(x)+\mathrm{c}_{1} u_{2}(x)+\frac{\mathrm{c}_{2} u_{1}{ }^{\prime}(x)}{x}+\frac{\mathrm{c}_{1} u_{2}{ }^{\prime}(x)}{x}+$
$c_{2} u_{1}{ }^{\prime \prime}(x)+u_{2}{ }^{\prime \prime}(x)+\mathrm{c}_{1} u_{2}{ }^{\prime \prime}(x), u_{3}{ }^{\prime}(0)=0, u_{3}(1)=0$.

And has the following solution:

$$
\begin{aligned}
& u_{3}\left(x, c_{1}, c_{2}, c_{3}\right)=\frac{1}{720}\left(-1+x^{2}\right)\left(60\left(19+x^{2}\right)+\left(881+86 x^{2}+\right.\right. \\
& \left.\left.x^{4}\right) c_{1}{ }^{3}+60 c_{1}\left(6+\left(19+x^{2}\right) c_{2}\right)+360\left(c_{2}+c_{3}\right)\right) .
\end{aligned}
$$

Substituting eq. (19), (21), (23) and (25) yields the third-order OHAM approximation solution for $(m=3)$ for eq. (15) 
$\tilde{u}\left(x, \mathrm{c}_{1}, \mathrm{c}_{2}, \mathrm{c}_{3}\right)=$

$u_{0}(x)+u_{1}\left(x, \mathrm{c}_{1}\right)+u_{2}\left(x, \mathrm{c}_{1}, \mathrm{c}_{2}\right)+u_{3}\left(x, \mathrm{c}_{1}, \mathrm{c}_{2}, \mathrm{c}_{3}\right) .($

On the domain between $a=0$ and $b=1$, the residual is

$R=x \tilde{u}^{\prime \prime}\left(x, \mathrm{c}_{1}, \mathrm{c}_{2}, \mathrm{c}_{3}\right)+\tilde{u}^{\prime}\left(x, \mathrm{c}_{1}, \mathrm{c}_{2}, \mathrm{c}_{3}\right)+x \tilde{u}\left(x, \mathrm{c}_{1}, \mathrm{c}_{2}, \mathrm{c}_{3}\right)$.

The less square method can be applied as

$\mathrm{J}\left(\mathrm{c}_{1}, \mathrm{c}_{2}, \mathrm{c}_{3}\right)=\int R^{2} \mathrm{dx}$

Thus, the following values of convergent control parameters $c_{i}{ }^{\prime} s, i=1,2,3$ are obtained by applying the condition (14) as follow:

$c_{1}=-0.7308282927162905, c_{2}=0.004724683876999099$,

$c_{3}=-0.0014964083830838778$

By using these values of the convergent control parameters, the third-order approximate solution in (12) become:

$$
\begin{aligned}
\tilde{u}\left(x, c_{1}, c_{2}, c_{3}\right)= & 1.3068495266302014-0.3267012651195327 x^{2} \\
& -0.020393881120754326 x^{4}-0.0005421426314229495 x^{6} .
\end{aligned}
$$


Table 1: comparison between the OHAM solution and spline solution together with the exact solution for example 1

\begin{tabular}{cccccc}
\hline$x$ & Exact & $\begin{array}{c}\text { Spline } \\
h=1 / 40\end{array}$ & OHAM & $\mid$ Error-Spline & $\mid$ Error-OHAM $\mid$ \\
\hline 0.000 & 1.306956 & 1.306840 & 1.306849 & 0.000116 & 0.000107 \\
0.025 & 1.306752 & 1.306636 & 1.306645 & 0.000116 & 0.000107 \\
0.050 & 1.306139 & 1.306023 & 1.306032 & 0.000116 & 0.000106 \\
0.075 & 1.305119 & 1.305003 & 1.305012 & 0.000116 & 0.000106 \\
0.100 & 1.303691 & 1.303575 & 1.303584 & 0.000116 & 0.000106 \\
0.200 & 1.293919 & 1.293804 & 1.293814 & 0.000115 & 0.000105 \\
0.300 & 1.277714 & 1.277601 & 1.277611 & 0.000113 & 0.000102 \\
0.400 & 1.255198 & 1.255088 & 1.255097 & 0.00011 & 0.000101 \\
0.500 & 1.226539 & 1.226432 & 1.226440 & 0.000107 & 0.000099 \\
0.600 & 1.191950 & 1.191848 & 1.191854 & 0.000102 & 0.000096 \\
0.700 & 1.151690 & 1.151594 & 1.151598 & 0.000096 & 0.000092 \\
0.800 & 1.106059 & 1.105969 & 1.105971 & 0.000089 & 0.000087 \\
0.900 & 1.05539 & 1.055312 & 1.055313 & 0.000078 & 0.000077 \\
1.00 & 1.000071 & 1.000000 & 1.000000 & 0.000070 & 0.000070 \\
\hline & & & & & \\
\hline
\end{tabular}

From this table it can be seen that the result obtained by using three order OHAM solutions is nearly identity to the exact solution.

Example 2: consider the following example:

$$
\begin{aligned}
& u^{(4)}(x)=1-4 u(x) \quad,-1 \leq x \leq 1 \\
& u(-1)=0, u^{\mathrm{m}}(-1)=0, u(1)=0, u^{\mathrm{m}}(1)=0 .
\end{aligned}
$$

The exact solution of this problem in the case is given by 


$$
u(x)=\frac{1}{4}\left[1-\frac{2(\operatorname{Sin}(1) \operatorname{Sinh}(1) \operatorname{Sin} x \operatorname{Sinh} x+\operatorname{Cos}(1) \operatorname{Cosh}(1) \operatorname{Cos} x \operatorname{Cosh} x)}{\operatorname{Cos}(2)+\operatorname{Cosh}(2)}\right]
$$

To use the basic ideas of OHAM formulated according to eq. (1), we define the linear and nonlinear operators in the following form

$$
\begin{aligned}
& L[v(x ; q)]=\left(\frac{\mathrm{d}^{4} v(x ; q)}{\mathrm{d} x^{4}}\right) \\
& N[v(x ; q)]=\frac{\mathrm{d}^{4} v(x ; q)}{\mathrm{d} x^{4}}+4 v(x ; q)-1
\end{aligned}
$$

Now, apply eq. (30) when $q=0$, it gives the zeroth-order problem as follow:

$$
u_{0}{ }^{(4)}=0, u_{0}(1)=0, u_{0}{ }^{s}(1)=0, u_{0}{ }^{s}(-1)=0, u_{0}(-1)=0 \text {. }
$$

The solution of eq. (3.1.6) is given by

$u_{0}(x)=0$.

From eq. (6) the first-order problem is

$$
u_{1}{ }^{(4)}\left(x, c_{1}\right)=-c_{1}, u_{1}{ }^{\prime \prime}(-1)=u_{1}(-1)=u_{1}{ }^{n g}(1)=u_{1}(1)=0 \text {. }
$$

which has the following solution

$u_{1}\left(x, c_{1}\right)=-\frac{5 c_{1}}{24}+\frac{x^{2} c_{1}}{4}-\frac{x^{4} c_{1}}{24}$

From eq. (7), the second-order problem is

$$
\begin{aligned}
& u_{2}{ }^{(4)}\left(x, c_{1}, c_{2}\right)=-c_{1}-\frac{11 c_{1}^{2}}{6}+x^{2} c_{1}^{2}-\frac{1}{6} x^{4} c_{1}^{2}- \\
& c_{2}, u_{2}{ }^{\prime \prime}(-1)=u_{2}(-1)=u_{2}{ }^{s}(1)=u_{2}(1)=0 .
\end{aligned}
$$


And has the following solution

$$
\begin{aligned}
u_{2}\left(x, c_{1}, c_{2}\right)= & -\frac{5 c_{1}}{24}+\frac{x^{2} c_{1}}{4}-\frac{x^{4} c_{1}}{4}-\frac{697 c_{1}^{2}}{2016}+\frac{151 x^{2} c_{1}^{2}}{360}-\frac{11 x^{4} c_{1}^{2}}{144}+\frac{x^{6} c_{1}^{2}}{360} \\
& -\frac{x^{8} c_{1}^{2}}{10080}-\frac{5 c_{2}}{24}+\frac{x^{2} c_{2}}{4}-\frac{x^{4} c_{2}}{24} .
\end{aligned}
$$

When $\mathrm{k}=3$, and by applying eq. (8), the third-order problem become

$u_{3}(-1)=u_{3}{ }^{\mathrm{I}}(-1)=u_{3}(1)=u_{3}{ }^{\mathrm{I}}(1)=0$.

The solution of equation (38) is given below

$$
\begin{aligned}
& u_{3}\left(\mathrm{x}, \mathrm{c}_{1}, \mathrm{c}_{2}, \mathrm{c}_{3}\right)=-\frac{5 c_{1}}{24}+\frac{x^{2} c_{1}}{4}-\frac{x^{4} c_{1}}{24}-\frac{697 c_{1}^{2}}{1008}+\frac{151}{180} x^{2} c_{1}^{2}- \\
& \frac{11}{72} x^{4} c_{1}^{2}+\frac{1}{180} x^{6} c_{1}^{2}-\frac{x^{8} c_{1}^{2}}{5040}-\frac{3433333 c_{1}^{3}}{5987520}+\frac{317641 x^{2} c_{1}^{3}}{453600}- \\
& \frac{1621 x^{4} c_{1}^{3}}{241 x^{6} c_{1}^{3}}-\frac{17 x^{8} c_{1}^{3}}{60480}+\frac{x^{10} c_{1}^{3}}{453600}-\frac{x^{12} c_{1}^{3}}{29937600}-\frac{5 c_{2}}{24}+ \\
& \frac{x^{2} c_{2}}{4}-\frac{x^{4} c_{2}}{24}-\frac{697 c_{1} c_{2}}{1008}+\frac{151}{180} x^{2} c_{1} c_{2}-\frac{11}{72} x^{4} c_{1} c_{2}+ \\
& \frac{1}{180} x^{6} c_{1} c_{2}-\frac{x^{8} c_{1} c_{2}}{5040}-\frac{5 c_{3}}{24}+\frac{x^{2} c_{3}}{4}-\frac{x^{4} c_{3}}{24}
\end{aligned}
$$

By substitution these values of the convergent control parameters in eq. (40)

third order approximation become

$$
\tilde{u}\left(x, c_{1}, c_{2}, c_{3}\right)=u_{0}(x)+u_{1}\left(x, c_{1}\right)+u_{2}\left(x, c_{1}, c_{2}\right)+u_{3}\left(x, c_{1}, c_{2}, c_{3}\right) .
$$

On the domain between $a=0$ and $b=1$, the residual is

$$
R=u^{4}\left(x, c_{1}, c_{2}, c_{3}\right)-1+4 u\left(x, c_{1}, c_{2}, c_{3}\right), \quad-1 \leq x \leq 1,
$$


the less square method can be applied as

$J\left(c_{1}, c_{2}, c_{3}\right)=\int R^{2} d x$

And

$\frac{d j}{d c_{1}}=\frac{d j}{d c_{2}}=\frac{d j}{d c_{3}}=0$

Thus, the values of the convergent control parameters are obtained in the following form

$c_{1}=-0.8427030133903135, c_{2}=-0.03745389496031039, c_{3}=$ 0.007891985031813164

The approximate solution (40) now become

$\tilde{u}\left(x, c_{1}, c_{2}, c_{3}\right)=$

$0.1254157425664142-0.14777096526408612 x^{2}+$

$0.020764064567026067 x^{4}+$

$0.0016418600724808347 x^{6}-$

$0.00004940261018944155 x^{8}-$

$0.000001319321363145261 x^{10}+$

$1.998971762341304 \times 10^{-8} x^{12}$ 
Table 2: Exact and approximate solution using OHAM for Example 2

\begin{tabular}{lccc}
\hline$x$ & Exact & OHAM & $\mid$ Error-OHAM $\mid$ \\
\hline 0.0 & 0.1254157423 & 0.1254157425 & $2.05210 \times 10^{-10}$ \\
0.1 & 0.1239401108 & 0.1239401109 & $1.58996 \times 10^{-10}$ \\
0.2 & 0.1195382313 & 0.1195382314 & $4.40237 \times 10^{-11}$ \\
0.3 & 0.1122857383 & 0.1122857382 & $8.11986 \times 10^{-11}$ \\
0.4 & 0.1023106408 & 0.1023106407 & $1.54609 \times 10^{-10}$ \\
0.5 & 0.0897962152 & 0.0897962150 & $1.44571 \times 10^{-10}$ \\
0.6 & 0.0749849828 & 0.0749849827 & $6.76991 \times 10^{-11}$ \\
0.7 & 0.0581836997 & 0.0581836997 & $2.16225 \times 10^{-11}$ \\
0.8 & 0.0397692606 & 0.0397692607 & $6.71113 \times 10^{-11}$ \\
0.9 & 0.0201953945 & 0.0201953946 & $5.03632 \times 10^{-11}$ \\
1.0 & 0 & 0 & $1.1418004128 \times 10^{-17}$ \\
\hline
\end{tabular}




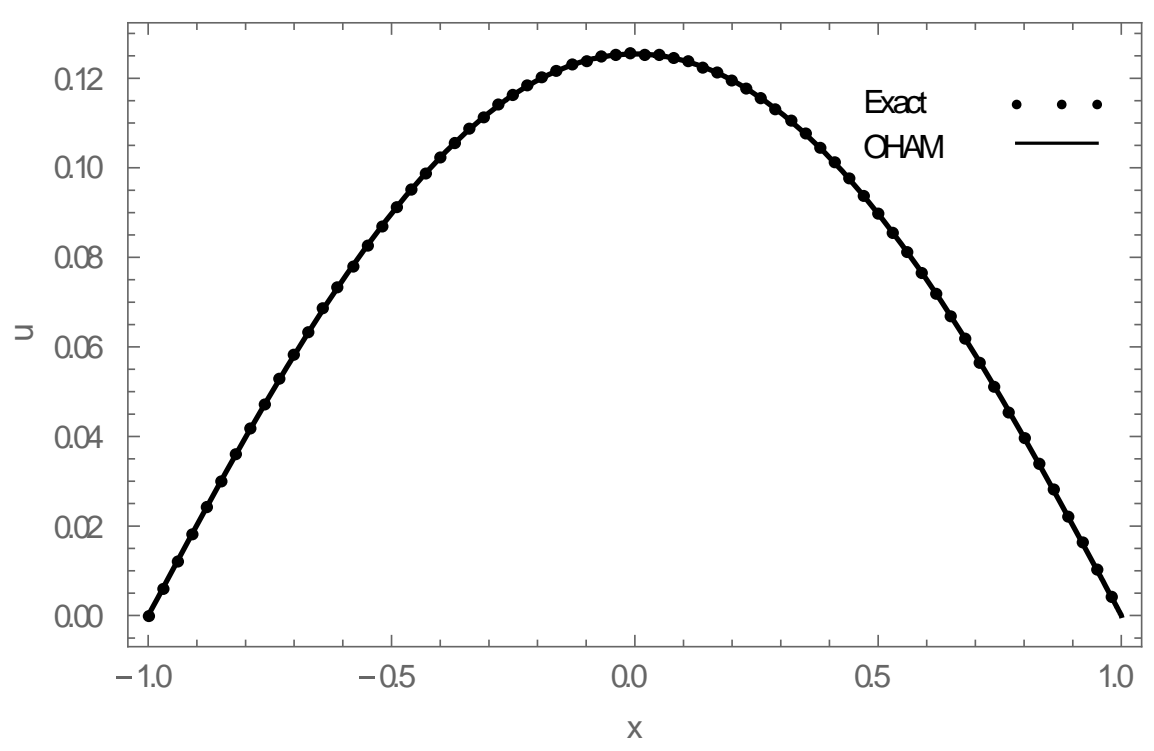

Figure 1 : Exact and approximate solution using OHAM for example 2.

Example 2 is regulated in Table 2 and Fig. 1 which show high accuracy of OHAM, that proves and demonstrate the capability and reliability of the OHAM.

\section{Conclusion}

OHAM has been applied successfully to obtain approximate analytical solution of singular boundary value problem and higher order boundary value problem. The practicality and effectively of OHAM have been illustrated through two examples. This shows that the method is efficient and reliable from singular two points boundary value problems and higher-order boundary value problem.

\section{References}

[1] N. Anakira, A. Ratib, K. Alomari, and Ishak Hashim, " Application of optimal homotopy asymptotic method for solving linear delay differential equations", The 2013 UKM FST Postgraduate Colloquium. AIP Conf. Proc., 1571, (2013). 1013-1019.

[2] M. Esmaeilpour and D. D, Ganji," Solution of the Jeffery-Hamel flow problem by optimal homotopy asymptotic method", Computers and Mathematics with Applications, 59,(2010), 3405-3411. 
[3] A. Golbabai, M. Fardi, and K. Sayevand," Application of the optimal homotopy asymptotic method for solving a strongly nonlinear oscillatory system.", Mathematical and Computer Modelling, Vol.58, (2013), 1837-1843.

[4] S. Haq, and M. Ishaq," Solution of strongly nonlinear ordinary differential equations arising in heat transfer with optimal homotopy asymptotic method.", InternationalJournal of heat and Mass Transfer, Vol.55, (2012), 5737-5743.

[5] N. Khan, T. Mahmood, and M. S. Hashmi," OHAM solution for thin film flow of a third order fluid through porous medium over an inclined plane.", Heat Transfer Research, Vol..44, (2013), 719-731.

[6] S. Iqbal, and A. Javed," Application of Optimal Homotopy Asymptotic Method for the Analytic Solution of Singular Lane-Emden type Equation.", Heat Transfer Research, Vol. 217, (2011), 7753-7761.

[7] F. E. Mabood, W.A. Khan, and A.I. Ismail," Solution of nonlinear boundary layer equation for flat plate via optimal homotopy asymptotic method.", Heat Transfer-Asian Research, Vol. 43, (2014b), 197-203.

[8] V. Marinca, and N. Herisanu," Application of optimal homotopy asymptotic method for solving nonlinear equations arising in heat transfer.", International Communications in Heat and Mass Transfer, Vol.35, (2008), 710-715.

[9] V. Marinca, and N. Herisanu and L. Nemes," Optimal homotopy asymptotic method with application to thin film flow.", Central European Journal of Physics, Vol.6, (2008), 648-653.

[10] V. Marinca, N. Herisanu , C. Bota and B. Marinca," An optimal homotopy asymptotic method applied to the steady flow of a fourth-grade fluid past a porous plate.", Applied Mathematics Letters, Vol.22, (2009), 245-251.

[11] S. Nadeem, R. Mehmood, and N.S. Akbar," Optimized analytical solution for oblique flow of a Casson-nano fluid with convective boundary conditions.", Applied Mathematics Letters, Vol.22, (2014), 245-251. 